\title{
Avaliação das alterações odontológicas em sincrânios de Cerdocyon thous oriundos de atropelamentos na rodovia ES-060, Espírito Santo ${ }^{1}$
}

\author{
João Luiz Rossi Junior ${ }^{2 *}$, Ana Paula Airosa de Castro ${ }^{3}$ e Marina Drago Marchesi ${ }^{4}$
}

\begin{abstract}
Rossi Jr J.L., Castro A.P.A. \& Marchesi M.D. 2012. [Evaluation of dental changes in sincranium of Cerdocyon thous from running over in the ES-060 road, Espirito Santo.] Avaliação das alterações odontológicas em sincrânios de Cerdocyon thous oriundos de atropelamentos na rodovia ES-060, Espírito Santo. Pesquisa Veterinária Brasileira 33(6):785-790. Setor de Odontologia Veterinária, Universidade Vila Velha, Rua Comissário José Dantas de Melo 21, Boa Vista, ES 29102-770, Brazil. E-mail: joao.rossi@uvv.br

The crab-eating foxes (Cerdocyon thous Linnaeus, 1766) are animals widely found in South American countries. Although it is not an endangered species, it is possible that many of them suffer impacts from being hit by car on the highways of the country, and this is the reason why it is one of the carnivores' species with high frequency of deaths. 32 free living sincranium stored in the osteological collection in the Laboratory of Veterinary Anatomy of Vila Velha University (Vila Velha/ES) were studied in order to diagnose diseases suffered by these individuals while alive. The main lesions identified were: crowding, no dental, dental calculus, tertiary dentin, detrition, darkening tooth, exposure of pulp, bone fenestration, fractured tooth, enamel fracture, giroversion, pigmentation and resorption of the alveolar crest. The most common findings were: no dental, dental detrition and fractured tooth. No dental before death, bone changes, dental calculus, crowding and giroversion apparently did not cause any harm to the animals while alive.
\end{abstract}

INDEX TERMS: Oral cavity, crab-eating foxes, Cerdocyon thous, free living, postmortem, wild canids.

RESUMO.- Os cachorros-do-mato (Cerdocyon thous Linnaeus, 1766) são animais amplamente encontrados em países da América do Sul. Apesar de não ser uma espécie ameaçada de extinção, é possível que muitas populações sofram impactos decorrentes de atropelamentos de indivíduos nas rodovias do país, pois, trata-se de uma das espécies de carnívoros com elevada ocorrência de mortes deste tipo. Foram avaliados 32 sincrânios de $C$. thous, oriundos de vida livre, armazenados na coleção osteológica do Laboratório de Anatomia Veterinária da Universidade Vila Velha (Vila Velha/ES), a fim de diagnosticar doenças que acometeram estes indivíduos enquanto vivos. As lesões macroscópicas identificadas foram: apinhamento dentário, ausên-

\footnotetext{
${ }^{1}$ Recebido em 21 de setembro de 2012.

Aceito para publicação em 2 de maio de 2013.

${ }^{2}$ Setor de Odontologia Veterinária e Setor de Animais Selvagens, Universidade Vila Velha (UVV), Rua Comissário José Dantas de Melo 21, Boa Vista, ES 29102-770, Brasil. *Autor para correspondência: joao.rossi@uvv.br

${ }^{3}$ Mestre em Ciência Animal, subárea Manejo e Conservação de Animais Silvestres, UVV, Boa Vista, ES.

${ }^{4}$ Setor de Odontologia Veterinária e Setor de Animais Selvagens, UVV, Boa Vista, ES.
}

cia dentária, cálculo dentário, dentina terciária, desgaste, escurecimento dentário, exposição de polpa, fenestração óssea, fratura dentária, fratura de esmalte, giroversão, pigmentação e reabsorção da crista alveolar. Os achados mais comumente observados foram: ausência dentária, desgaste dentário e fratura dentária. Ausências dentárias anteriores à morte, alterações ósseas, cálculos dentários, apinhamento e giroversão aparentemente não causaram quaisquer prejuízos aos animais enquanto vivos.

TERMOS DE INDEXAÇÃO: Cavidade oral, pós-morte, cachorro-domato, Cerdocyon thous, canídeo selvagem, vida livre.

\section{INTRODUÇÃO}

A cavidade oral, os dentes e os tecidos associados, são estruturas fundamentalmente importantes para a sanidade de todos os animais, sejam eles domésticos ou selvagens (Pachaly 2006). A cavidade oral dá início ao trato digestório, portanto sua higidez é fundamental para a eficiência de todos os sistemas de processamento de alimentos e nutrientes no organismo. Assim, a prevenção de transtornos médicos capazes de acometer a cavidade oral preserva a 
eficiência dos mecanismos de digestão, contribuindo decisivamente para a qualidade de vida dos animais, mantendo sua sanidade geral e aumentando sua eficiência reprodutiva e sua expectativa de vida. Problemas de menor importância geram desconforto e dor, enquanto doenças orais severas levam à diminuição de ingestão de água e alimento causando debilidade, alterações sistêmicas e, por fim, até a morte do animal (Gioso \& Rossi Jr 2001, Pachaly 2006, Venturini 2006).

Geralmente, os dentes são estruturas que se encontram preservadas em crânios e fósseis, permitindo desta forma, a reconstrução de prováveis hábitos alimentares de diferentes espécies e o estudo de socialização e competitividade entre espécies e antigas comunidades (Van Valkenburgh 1989).

Pode-se afirmar que o exame pós-morte de crânios e outros ossos costumavam ser negligenciados na Medicina Veterinária. Felizmente, o número de estudos sobre enfermidades em animais silvestres, que vem utilizando este material, tem aumentado significativamente, associando-se a outros exames como avaliação radiográfica e microscópica. Além disso, a possibilidade de realização de estudos comparativos sobre a evolução dos animais, correlacionando com o tipo de dieta e de enfermidades dentárias existentes há séculos atrás e na atualidade, podem trazer respostas e fomentar a busca por inúmeras outras. (Berger et al. 2004, Cooper \& Cooper 2008).

A investigação osteológica pode revelar inúmeras informações úteis sobre a saúde anterior à morte de um espécime, bem como afecções infecciosas e físicas, tanto em humanos como em animais. Os acervos de história natural dos museus são fonte de conhecimento e compreensão sobre as espécies e seus hábitats. É possível obter informações sobre a alimentação dos animais durante a vida, o grau de poluição química que os circundava, enquanto forrageavam plantas, por exemplo, e possivelmente, outras informações que ainda não sabemos revelar (Elbroch 2006, Cooper \& Cooper 2008).

A literatura existente sobre enfermidades orais em animais silvestres é, em sua maioria, sobre animais mantidos em cativeiro, descrevendo grande ocorrência de doenças

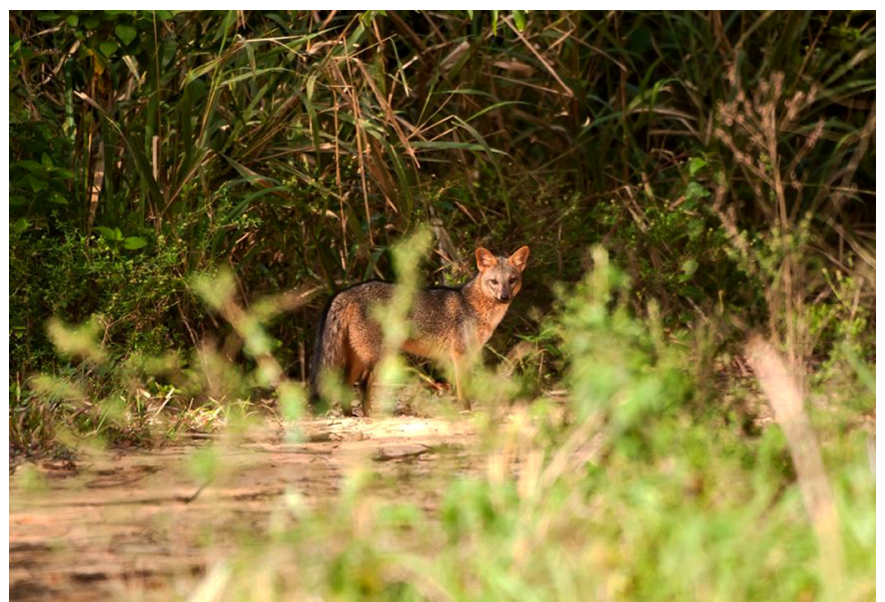

Fig.1. Cachorro-do-mato, Cerdocyon thous, observado em uma reserva, próximo à Rodovia ES-060. da cavidade oral devido a traumas e doença periodontal. As lesões traumáticas geralmente ocorrem em decorrência do estresse de captura destes animais, acidentes durante brincadeiras ou agressão, e problemas comportamentais, como morder grades de metal, paredes, portões e cercas (Wiggs \& Lobprise 1997, Wiggs \& Bloom 2003). Embora a ocorrência de fraturas dentárias em animais silvestres seja

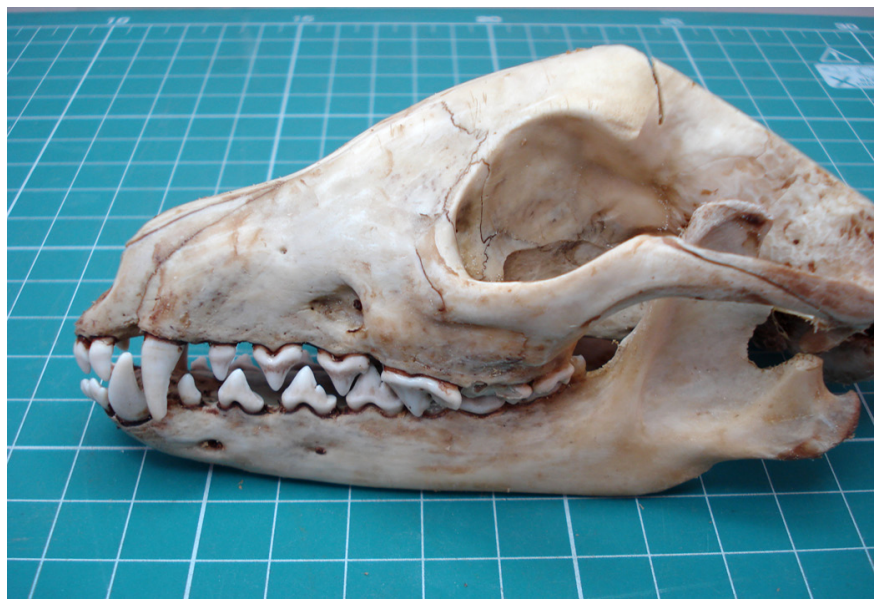

Fig.2. Sincrânio de cachorro-do-mato, Cerdocyon thous, disposto sobre base milimetrada para avaliação macroscópica de alterações dentárias.

Quadro 1. Dados referentes aos sincrânios de Cerdocyon thous, na Universidade Vila Velha

\begin{tabular}{lcclc}
\hline Instituição & $\begin{array}{c}\text { Número de } \\
\text { Registro }\end{array}$ & $\begin{array}{c}\text { Data da } \\
\text { Coleta }\end{array}$ & Localidade & Sexo \\
\hline UVV & 1 & NR $^{\mathrm{b}}$ & Espírito Santo & NR \\
UVV & 2 & NR & Espírito Santo & NR \\
UVV & 3 & NR & Espírito Santo & NR \\
UVV & 4 & NR & Espírito Santo & NR \\
UVV & 5 & NR & Espírito Santo & NR \\
UVV & SM-10 & 2004 & Espírito Santo & Fêmea \\
UVV & SM-11 & 2004 & Espírito Santo & Fêmea \\
UVV & SM-13 & 2004 & Espírito Santo & Fêmea \\
UVV & SM-14 & 2004 & Espírito Santo & Macho \\
UVV & SM-15 & 2004 & Espírito Santo & Macho \\
UVV & SM-55 & 2005 & Espírito Santo & Fêmea \\
UVV & SM-61 & 2006 & Espírito Santo & Fêmea \\
UVV & SM-62 & 2006 & Espírito Santo & Macho \\
UVV & SM-63 & 2006 & Espírito Santo & Macho \\
UVV & SM-69 & NR & Espírito Santo & NR \\
UVV & SM-70 & 2006 & Espírito Santo & Macho \\
UVV & SM-71 & 2005 & Espírito Santo & Fêmea \\
UVV & SM-75 & 2005 & Espírito Santo & Fêmea \\
UVV & SM-76 & 2006 & Espírito Santo & Fêmea \\
UVV & SM-77 & 2006 & Espírito Santo & Fêmea \\
UVV & SM-78 & 2006 & Espírito Santo & Macho \\
UVV & SM-80 & 2008 & Espírito Santo & Fêmea \\
UVV & SM-81 & 2006 & Espírito Santo & Macho \\
UVV & SM-87 & 2006 & Espírito Santo & Fêmea \\
UVV & SM-89 & NR & Espírito Santo & NR \\
UVV & SM-91 & 2007 & Espírito Santo & Macho \\
UVV & SM-92 & 2007 & Espírito Santo & Fêmea \\
UVV & SM-94 & 2007 & Espírito Santo & Fêmea \\
UVV & SM-95 & 2006 & Espírito Santo & Macho \\
UVV & SM-97 & 2007 & Espírito Santo & Macho \\
UVV & SM-103 & NR & Espírito Santo & NR \\
UVV & SM-104 & NR & Espírito Santo & NR \\
TVta & & & &
\end{tabular}

Total 32

a Centro Universitário Vila Velha, ${ }^{\text {b }}$ Não relatado. 
especialmente atribuída ao ambiente de cativeiro, um estudo realizado na região Central do Brasil em lobos guará de vida livre, Furtado et al. (2006) constataram alta prevalência de fraturas dentárias nos animais de vida livre $(63,1 \%)$.

O C. thous (Fig.1) é uma espécie onívora, generalista e oportunista, e sua dieta varia sazonalmente, sendo constituída por frutos, pequenos vertebrados, insetos, crustáceos e peixes, podendo também alimentar-se de carniça. Em decorrência do elevado consumo de frutos, os cachorros-do-mato podem agir como dispersores de sementes (Santiago \& Oliveira 2001, Cheida et al. 2006, Gomes 2006).

Objetivou-se avaliar sincrânios (Fig.2) de cachorros-do-mato (Cerdocyon thous) e descrever possíveis lesões macroscópicas de animais provenientes de vida livre, relatan-

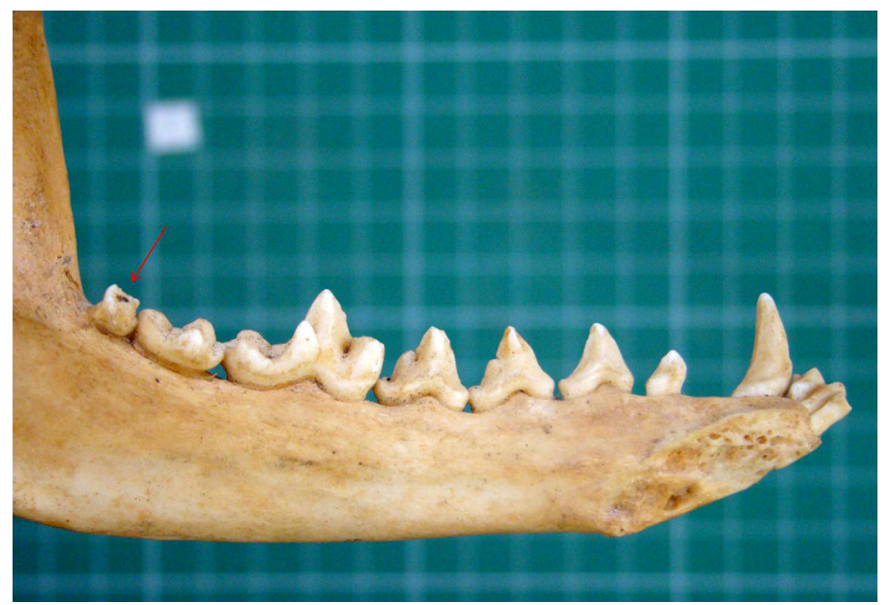

Fig.3. Apinhamento dentário de terceiro molar inferior esquerdo de cachorro-do-mato, Cerdocyon thous, apontado pela seta.

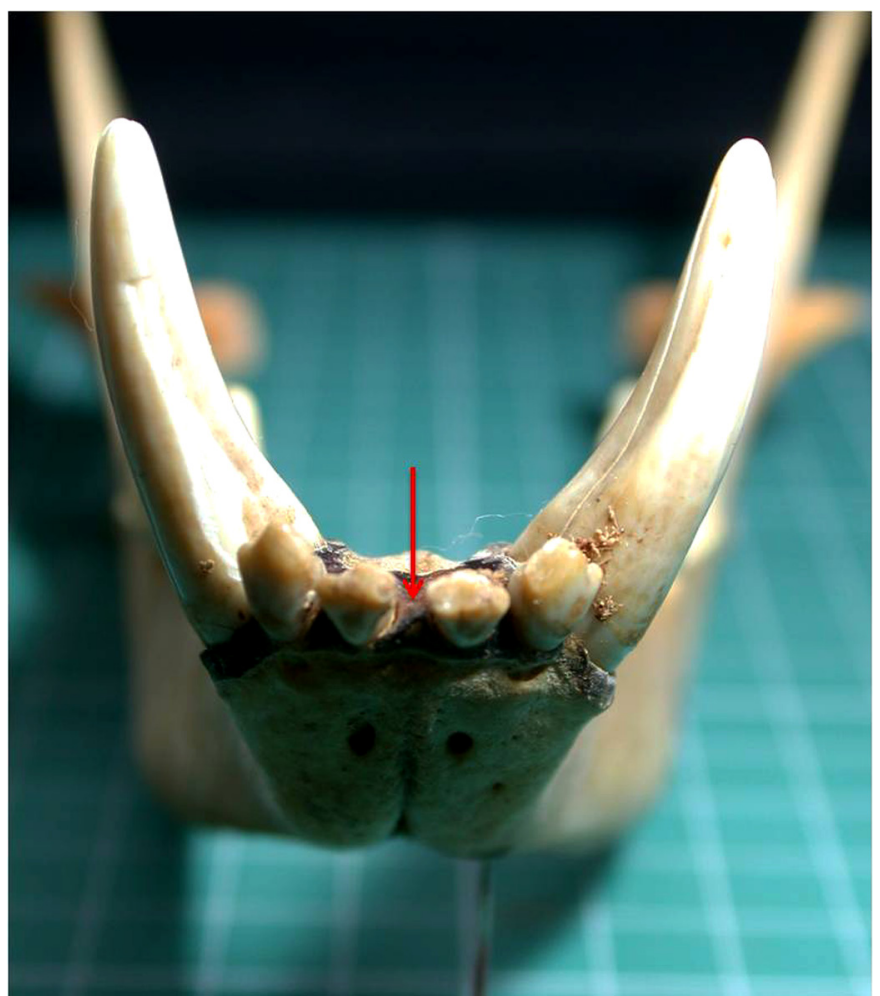

Fig.4. Ausência dentária antes da morte de incisivos inferiores de cachorro-do-mato, Cerdocyon thous, apontado pela seta. do a prevalência diferenciada dos vários tipos de afecções odontológicas anteriores e posteriores à morte, buscando possíveis alterações dentárias, e se essas foram motivo para dificuldade ou incapacidade de alimentação.

\section{MATERIAL E MÉTODOS}

Os Cerdocyon thous recolhidos da rodovia por funcionários da RODOSOL eram encaminhados ao Setor de Patologia Veterinária da Universidade Vila Velha (UVV). Os sincrânios estudados foram preparados através de diversos métodos de maceração e/ou complementada por insetos necrófagos, conforme técnicas específicas escolhidas segundo a conveniência da instituição, configurando diferenças importantes e notórias em cada peça estudada. Foram avaliados sincrânios de 32 indivíduos de $C$. thous provenientes de vida livre e que foram a óbito devido a atropelamento. A identificação dos sincrânios, bem como data de coleta, localidade e sexo, são apresentadas no Quadro 1.

As peças foram avaliadas quanto à presença de assimetria, fraturas, lesões ósseas e oclusão. Os dentes e osso alveolar foram inspecionados macroscópica e visualmente. Os achados observados nos sincrânios foram: apinhamento dentário (Fig.3), ausência dentária antes da morte (Fig.4), cálculo dentário, dentina terciária, desgaste dentário (Fig.5), escurecimento dentário, exposição de polpa dentária, fenestração óssea alveolar, fratura dentária, fratura de esmalte, giroversão dentária (Fig.6), pigmentação de

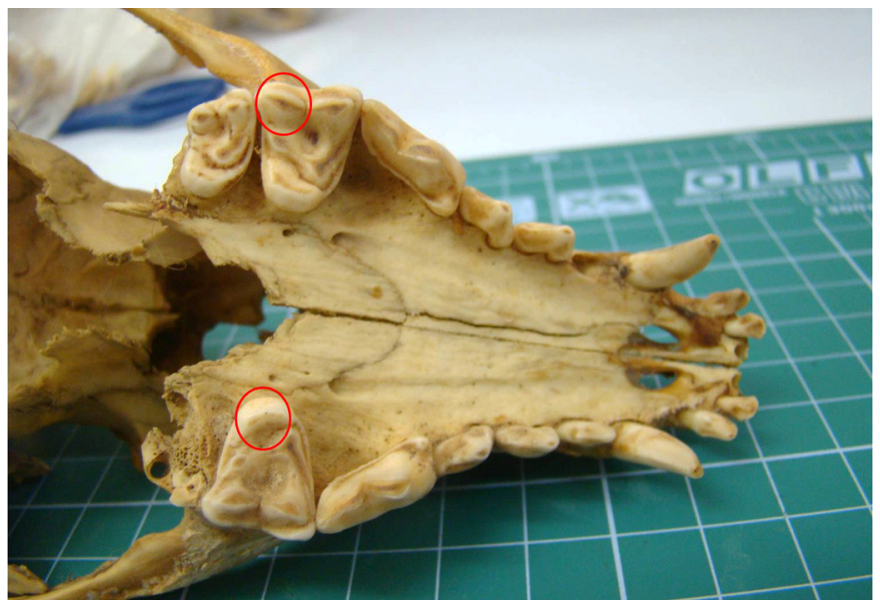

Fig.5. Desgaste dentário nas cúspides dos molares superiores de cachorro-do-mato, Cerdocyon thous.

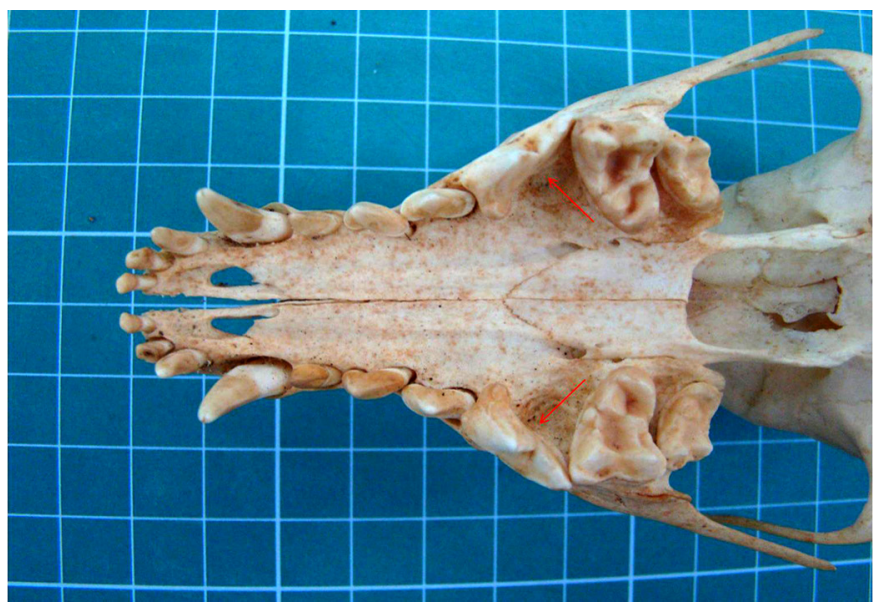

Fig.6. Giroversão de quarto pré-molar superior esquerdo e direito de cachorro-do-mato, Cerdocyon thous, apontados pelas setas. 


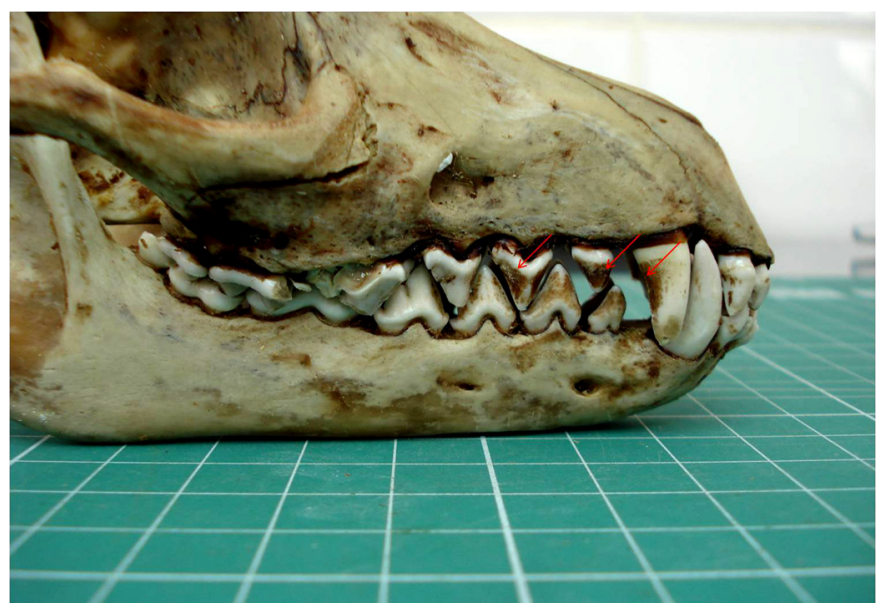

Fig.7. Pigmentação de esmalte dentário de cachorro-do-mato, Cerdocyon thous, apontado pela seta.

esmalte (Fig.7), reabsorção de crista alveolar (medida em milímetros).

A reabsorção óssea foi mensurada em milímetros, tomando-se a medida da distância entre a junção amelo-cementária (JAC) e a crista alveolar, nas faces ósseas mesial, vestibular, distal e palatina ou lingual. Foi estabelecida a subtração padrão de $1,5 \mathrm{~mm}$ a partir da JAC, a fim de caracterizar a medida do espaço biológico, ocupado pelo epitélio juncional e inserção conjuntiva. Em dentes multi-radiculares foram realizadas medidas para cada raiz.

Para determinação de fenestração e deiscência ósseas, foi adotada metodologia descrita por Davies et al. (1974), isto é, considerou-se deiscência o defeito ósseo vertical apresentando medida de crista óssea igual ou superior a $4 \mathrm{~mm}$, comparada à crista alveolar adjacente, com base na JAC e fenestração, o defeito ósseo localizado na tábua óssea alveolar causando exposição radicular, sem comprometimento da margem da crista alveolar.

A ausência dentária foi distinguida entre antes e após a morte do animal, de acordo com a forma e o padrão da margem óssea alveolar. Considerou-se a perda dentária anterior à morte na presença de osso alveolar com as seguintes características: contorno irregular ou arredondado da crista óssea alveolar, diminuição da profundidade do alvéolo, reação periosteal exuberante e aumento da vascularização, evidenciada pela presença de grande número de foraminas (Verstraete et al. 1996).

Os sincrânios foram avaliados macroscopicamente, por meio de inspeção visual direta. As medições e aferições foram realizadas através da utilização de sonda periodontal milimetrada de uso odontológico. Para avaliação odontológica, os sincrânios foram dispostos em bancada de trabalho, sobre base milimetrada utilizada como fundo.

Foram feitas documentações fotográficas através da utilização de câmera digital em todos os sincrânios estudados. Para cada espécime, foram realizados registros fotográficos da vista lateral (esquerda e direita), frontal, dorsal e ventral da cavidade oral e das afecções presentes.

Os resultados encontrados foram catalogados individualmente em fichas odontológicas, denominadas odontogramas, conforme o modelo utilizado por Lopes (2008) modificadas e adaptadas especialmente para serem utilizados em sincrânios da espécie estudada.

A avaliação estatística se deu através de estatística descritiva, com o intuito de demonstrar o percentual dos resultados obtidos na avaliação dos sincrânios de $C$. thous.
Quadro 2. Lesões observadas nos sincrânios de Cerdocyon thous tombados na coleção osteológica da Universidade Vila Velha

\begin{tabular}{lcc}
\hline \multicolumn{1}{c}{ Lesão } & $\begin{array}{c}\text { Número de } \\
\text { sincrânios }\end{array}$ & Porcentagem \\
\hline Apinhamento dentário & 2 & $6,45 \%$ \\
Ausência dentária antes da morte & 4 & $12,90 \%$ \\
Ausência dentária após a morte & 21 & $67,74 \%$ \\
Cálculo dentário grau I & 8 & $25,80 \%$ \\
Dentina terciária reparadora & 10 & $32,25 \%$ \\
Desgaste dentário grau I & 21 & $67,74 \%$ \\
Desgaste dentário grau II & 10 & $32,25 \%$ \\
Escurecimento dentário & 8 & $25,80 \%$ \\
Exposição de polpa & 12 & $38,70 \%$ \\
Fenestração de osso alveolar & 5 & $16,12 \%$ \\
Fratura dentária & 14 & $45,16 \%$ \\
Fratura de esmalte & 10 & $32,25 \%$ \\
Giroversão & 4 & $12,90 \%$ \\
Pigmentação de esmalte & 15 & $48,38 \%$ \\
Reabsorção de crista alveolar & 2 & $6,45 \%$
\end{tabular}

\section{RESULTADOS}

Dos 32 sincrânios avaliados de Cerdocyon thous, 10 pertenciam a machos (31\%), 13 pertenciam a fêmeas (41\%) e nove $(28 \%)$ não continham informações relacionadas ao sexo. Os sincrânios foram coletados entre os anos de 2004 a 2008, porém, nove peças não possuíam identificação relacionada ao ano de coleta. Em relação à idade estimada dos sincrânios, houve a identificação de apenas um (SM-103) filhote. Os outros 31 sincrânios eram pertencentes a animais adultos. Todos os 32 animais vieram a óbito devido a atropelamentos na Rodovia ES 0-60, trecho limitado à Concessionária RODOSOL, que liga as cidades de Guarapari a Vitória no Estado do Espírito Santo.

Ausência dentária pós-morte $(67,74 \%)$, desgaste dentário grau I $(67,74 \%)$, fratura dentária $(45,16 \%)$ e pigmentação de esmalte $(48,38 \%)$ foram as lesões encontradas mais frequentes, enquanto reabsorção da crista alveolar (6,45\%), apinhamento dentário $(6,45 \%)$, giroversão $(12,90 \%)$ e ausência dentária antes da morte $(12,90 \%)$ foram as lesões encontradas com menor frequência no presente estudo.

As lesões encontradas nos sincrânios estão listadas a seguir, como mostra o Quadro 2. A estatística descritiva foi realizada apenas com base nos sincrânios pertencentes a animais adultos, totalizando 31 animais, excluindo-se um sincrânio de filhote, a fim de evitar equívocos relacionados à descrição de lesões.

Os sincrânios encontravam-se armazenados individualmente em sacolas plásticas. Os dados referentes a cada animal encontravam-se arquivados em Livros de Registros, pertencentes ao Setor de Patologia do Hospital Veterinário Prof. Ricardo Alexandre Hippler.

\section{DISCUSSÃO}

A maior prevalência entre os animais foram de indivíduos adultos, contrastando com o baixo número de filhotes. Outra constatação foi a de que não houve diferenças significativas entre a proporção de machos e fêmeas. As principais circunstâncias relacionadas ao atropelamento da fauna são a disponibilidade de alimento ao longo da rodovia, que atraem os animais, e a fragmentação da área natural, que interfere no deslocamento dos espécimes (Forman \& 
Alexander 1998, Clevenger et al. 2003). Apesar de muitos cachorros-do-mato virem a óbito por atropelamento, não se sabe ao certo o impacto que os atropelamentos representam para estas populações.

As lesões traumáticas, representadas por $67,74 \%$ de desgaste dentário e $45,16 \%$ de fratura dentária, foram as alterações mais prevalentes. Estes dados concordam com Lopes (2008), que encontrou resultados similares em lobos-guará. Talvez, tal prevalência se deve à mesma razão referida por esta autora, que cita hábitos alimentares de variados componentes de consistência mais dura e rígida do que músculo e vísceras, como sendo naturais para essa espécie. 0 desgaste dentário nos cachorros-do-mato acometeu principalmente os dentes incisivos, similar a ursos pardos, de cativeiro e vida livre, como mencionado por Wenker et al. (1999). Embora o desgaste dentário seja considerado um processo fisiológico, utilizado inclusive para estimativa de idade em muitas espécies, é possível que o desgaste possa levar à perda acentuada da superfície dentária, comprometendo sua função. Alguns trabalhos relatam a exposição de câmara pulpar associada ao desgaste (Wenker et al. 1999). Entretanto, no presente trabalho, o desgaste máximo observado foi o desgaste grau II, não havendo nenhum animal com exposição de polpa decorrente desta afecção. Ainda, foi possível observar que a formação de dentina terciária reparadora foi diretamente proporcional ao nível de desgaste.

A ocorrência de fraturas dentárias foi semelhante à descrita em cachorro-selvagem africano de vida livre (48\%), relatada por Steenkamp \& Gorrel (1999). Esses achados também são compatíveis com outros achados em canídeos selvagens, como o lobo-guará, conforme relatos de Furtado et al. (2006) e Lopes (2008). Grande parte da literatura existente sobre odontologia em animais selvagens atribui ao ambiente de cativeiro a ocorrência de fraturas dentárias nos animais, geralmente relacionadas com problemas comportamentais e agressividade (Wiggs \& Lobprise 1997, Wenker et al. 1999, Pachaly \& Gioso 2001, Wiggs \& Bloom 2003). Entretanto, os achados desta pesquisa, somados às observações de Verstraete et al. (1996), Steenkamp \& Gorrel (1999), Furtado et al. (2006) e Lopes (2008), demonstram que esta afecção dentária também ocorre frequentemente em animais de vida livre e, nestas circunstâncias a causa esteja, provavelmente, relacionada às características e hábitos alimentares destes animais.

Foi proposto por Van Valkenburgh (1989) que aqueles animais que se alimentam de presas pequenas, invertebrados e vegetais provavelmente tenham uma tendência a apresentar baixa prevalência de fraturas dentárias, o que contrapõe os resultados obtidos no presente estudo, já que o cachorro-do-mato também costuma se alimentar com este tipo de dieta e apresenta alta prevalência de traumas dentários em natureza. A localização anatômica e o tamanho dentário podem ser fatores predisponentes a diferentes forças. 0 dente canino é, sem dúvida, o mais proeminente e vulnerável a traumas (Harvey \& Emily 1993); e, como consequência, é o mais frequentemente lesionado, conforme foi observado neste trabalho e em outros estudos (Van Valkenburggh 1998, Furtado et al. 2006, Lopes 2008).
É valido ressaltar que as fraturas dentárias também podem ser causadas no momento da morte, principalmente por traumas e atropelamentos, quando se observa fraturas, sem o acometimento ou remodelamento das estruturas adjacentes aos dentes fraturados. Outra alteração observada com frequência foi o escurecimento dentário, que também aconteceu possivelmente no momento do impacto. Rossi Jr (2002), descreveu que o escurecimento dentário interno pode ocorrer em traumatismos; resultantes do rompimento de vasos pulpares e penetração de substância nos túbulos dentários.

Nos casos apresentando ausência dentária anterior à morte, não foi possível a diferenciação entre agenesia dentária e dente não erupcionado, pois não foram realizados exames radiográficos nos sincrânios avaliados, já que se pretendia simular a mesma metodologia a ser empregada em estudos de campo com animais vivos ou em sincrânios de indivíduos tombados em coleções de museus de mastozoologia. Nestes dois casos, é muito difícil o acesso a exames radiográficos odontológicos. Foi observada a perda dentária após a morte na maioria dos sincrânios avaliados $(67,74 \%)$. Essa situação ocorre provavelmente pelo método de preparação dos sincrânios, porém, Elbroch (2006) afirma que frequentemente faltam dentes em crânios de animais com idades mais avançadas e que este achado pode ser diferenciado de perdas ocorridas anteriormente à morte.

Defeitos ósseos denominados fenestração e deiscência também apresentaram relevância nesta pesquisa, sendo observadas $16,12 \%$ de fenestração e $6,45 \%$ de reabsorção alveolar nos sincrânios. De acordo com Miles \& Grigson (2003), esses defeitos ósseos ocorrem na face vestibular, principalmente no osso maxilar, sendo caracterizado pela exposição de raízes dentárias, relato condizente com os achados observados no presente trabalho.

Apesar de não ter sido realizada a avaliação da dieta dos animais estudados, sabe-se que a dieta natural dos cachorros-do-mato e da maioria das espécies selvagens costuma ser mais abrasiva do que a comumente oferecida aos animais de cativeiro, e certamente este fator contribui para a deposição de placa bacteriana e cálculo sobre os dentes, conforme descrito por diversos autores (Wiggs \& Lobprise 1997, Wenker et al. 1999, Miles \& Grigson 2003, Wiggs \& Bloom 2003). Entretanto, os resultados observados no presente trabalho contrastam estas afirmações, já que foi observada a presença de cálculo dentário em cachorros-do-mato de vida livre. Uma sugestão para justificar esse fato seria a perda de hábitos naturais e a maior proximidade com humanos em áreas peri-urbanas, podendo levar a doenças orais semelhantes às observadas em cães domésticos.

Outro achado importante foi a ocorrência de pigmentação do esmalte. Segundo Miles (1963) e Rossi Jr et al (2007), a dieta pode influenciar na pigmentação superficial do esmalte. A ocorrência deste achado está provavelmente relacionada à ingestão de peixes, crustáceos e vegetais, encontrados na região de manguezais presentes nessa região. 0 apinhamento dentário também foi observado, acometendo $6,45 \%$ dos sincrânios. A literatura aponta que essa alteração não se refere apenas à discrepância de tamanho entre dentes e arcos, pois, uma vez que se trata de uma alteração 
de etiologia multifatorial, muitas vertentes podem estar associadas com o apinhamento (Bernabé \& Flores-Mir 2006). Giroversão foi relatada em 12,90\% dos sincrânios. Trabalhos recentes também relatam a ocorrência dessa alteração em outros animais selvagens como macaco-prego (Fecchio 2005), onça-pintada e puma (Rossi Jr et al 2007) e lobo-guará (Lopes 2008).

\section{CONCLUSÕES}

Os resultados do presente trabalho permitem afirmar que o desgaste dentário consiste no principal achado odontológico e fraturas dentárias são frequentes em Cerdocyon thous.

Ausência dentária antes da morte, alterações de osso alveolar, cálculo dentário, apinhamento dentário e giroversão não causam prejuízos importantes aos animais.

Não foram observadas lesões dentárias que justificassem incapacidade de predação, alteração de hábitos alimentares ou morte dos animais.

Agradecimentos.- À Sinhá Laurinha, OSCIP responsável pelo monitoramento ambiental da Rodovia ES-60, à Universidade Vila Velha por ceder espaço para pesquisa, à Funadesp pela concessão de bolsa de pesquisa (Proc. 22/09) e ao CNPq pelos auspícios de financiamento de pesquisa, Proc. 474411/2010-9.

\section{REFERÊNCIAS}

Berger M., Berger M., Stich H., Hüster H., Roux P. \& Schawalder P. 2004. Feline dental resorptive lesions in the $13^{\text {th }}$ to $14^{\text {th }}$ Centuries. J. Vet. Dentistry 21:206-213.

Bernabé E. \& Flores-Mir C. 2006. Dental morphology and crowding: a multivariate approach. Angle Orthodontist 76:20-25

Cheida C.C., Oliveira E.N., Costa R.F., Mendes F.R. \& Quadros J. 2006. Ordem Carnívora, p.231-275. In: Reis N.R., Peracchi A.L., Pedro W.A. \& Lima I. (Eds), Mamíferos do Brasil. Vol.1. Londrina, PR. 437p.

Clevenger A.P., Chruszcz B. \& Gunson K. 2003. Spatial patterns and factors influencing small vertebrate fauna road-kill aggregations. Biological Conservation 109:15-26

Cooper J.E. \& Cooper M.E. 2008. Skeletal pathology of primates and other wildlife. Vet. Rec. 162:63-64.

Davies R.M., Downer M.C., Hull P.S. \& Lennon M.A. 1974. Alveolar defects in human skulls. J. Clin. Periodontol. 1:107-111.

Elbroch M. 2006. Animal skulls: a guide to North American species. Stackpole Books, Mechanicsburg. 726p.

Fecchio R.S. 2005. Prevalência de lesões orais em macacos-prego (Cebus apela) mantidos em cativeiro no Estado de São Paulo. Trabalho de Conclusão de Curso em Medicina Veterinária, Universidade Metodista de São Paulo, São Bernardo do Campo, SP. 63p.

Forman T.T.R. \& Alexander L.E. 1998. Roads and their major ecological effects. Annual Review of Ecology and Systematics 29:207-231.

Furtado M.M., Kashivakura C.K., Ferro C., Almeida J.A., Silveira L. \& Astete S. 2006. Immobilization of free-ranging maned wolf (Chrysocyon brachyurus) with tiletamine and zolazepam in central Brazil. J. Zoo Wildl. Med. 37:68-70.
Gioso M.A. \& Rossi Júnior J.L. 2001. Prevalence of oral lesions in Puma concolor and Panthera onca in the State of São Paulo, Brazil. Anais 15응 Annual Veterinary Dental Forum, Texas, SA. (Resumo)

Gomes M.S. 2006. Carnívora - Canidae (Lobo-guará, cachorro-do-mato, raposa do mato), p.492-504. In: Cubas Z.S., Silva J.C.R., Catão-Dias J.L. (Eds), Tratado de Animais Selvagens: medicina veterinária. Roca, São Paulo. 1376p.

Harvey C.E. \& Emily P. 1993. Small animal dentistry. Mosby, St Louis. 413p.

Lopes F.M. 2008. Avaliação do sistema estomatognático e de sincrânios de lobo-guará (Chrysocyon brachyurus) em vida livre e cativeiro. Dissertação de Mestrado em Cirurgia, Faculdade de Medicina Veterinária e Zootecnia, Universidade de São Paulo, SP. 151p.

Miles A.E.W. 1963. Pigmented enamel. Proc. Royal Soc. Med. 56:918-920.

Miles A.E.W. \& Grigson C. 2003. Colyer's variations and diseases of the teeth of animals. Cambridge University Press, Cambridge. 672p.

Pachaly J.R. \& Gioso M.A. 2001. The oral cavity, p.1-15. In: Fowler M.E. \& Cubas Z.S. (Eds), Biology, Medicine and Surgery of South American Wild Animals. Iowa State University Press, Ames. 550p.

Pachaly J.R. 2006. Odontoestomatologia, p.1068-1091. In: Cubas Z.S., Silva J.C.R., Catão-Dias J.L. (Ed.), Tratado de Animais Selvagens: medicina veterinária. Roca, São Paulo.

Rossi Jr J.L. 2002. Estudo comparativo entre os achados clínicos de lesões orais em onça-pintada (Panthera onca) e suçuarana (Puma concolor) mantidas em cativeiro no estado de São Paulo e indivíduos de vida livre no Pantanal Sul Mato-Grossense. Dissertação de Mestrado em Medicina Veterinária, Faculdade de Medicina Veterinária e Zootecnia, Universidade de São Paulo, SP. 97p.

Rossi Jr J.L., Gioso M.A., Teles M.Q. \& Domingues-Falqueiro L.M. 2007. Acompanhamento do crescimento dental em Puma concolor mantido em cativeiro. Pesq. Vet. Bras. 27:205-208.

Santiago M.E.B. \& Oliveira L.T.F. 2001. Order Carnivora, Family Canidae (dogs, foxes, maned wolves) Medicine, p.279-290. In: Fowler M.E. \& Cubas Z.S. (Eds), Biology, Medicine, and Surgery of South American Wild Animal. Wiley, Iowa. 550p.

Steenkamp G. \& Gorre C. 1999. Oral and dental conditions in adult African wild dog skulls: a preliminary report. J. Vet. Dentistry 16:65-68.

Van Valkenburgh B. 1989. Carnivore dental adaptations and diet: a study of trophic diversity within guilds, p.410-436. In: Gittleman J.L. (Ed.), Carnivore Behavior, Ecology, and Evolution. Cornell University Press, New York. 624p.

Venturini M. 2006. Estudo retrospectivo de 3055 animais atendidos no OdontoVet ${ }^{\circledR}$ (Centro Odontológico Veterinário) durante 44 meses. Dissertação de Mestrado em Cirurgia, Faculdade de Medicina Veterinária e Zootecnia, Universidade de São Paulo, SP. 103p

Verstraete F.J.M., Van Aarde R.J., Nieuwoudt B.A., Mauer E. \& Kass P.H. 1996. The dental pathology of feral cats on Marion Island. 2. Periodontitis, external odontoclastic resorption lesions and mandibular thickening. J. Comp. Pathol. 115:283-297.

Wenker C.J., Stick H., Muller M. \& Lussi A. 1999. A retrospective study of dental conditions of captive brown bears (Ursus arctos spp.) compared with free-ranging Alaskan grizzlies (Ursus arctos horribilis). J. Zoo Wildl. Med. 30:208-221.

Wiggs R.B. \& Lobprise H.B. 1997. Veterinary dentistry: principles and practice. Lippincott-Raven, Philadelphia. 748p.

Wiggs R.B. \& Bloom B.C. 2003. Exotic placental carnivore dentistry. Vet. Clin. North Am., Exotic Anim. Pract. 6:571-599. 